\title{
Spontaneous postpartum rupture of an ovarian artery aneurysm treated with embolization: A case report and review of the literature.
}

\author{
Mami Shibahara ${ }^{1}$, Emi Kondo ${ }^{1}$, Eiji Shibata ${ }^{1}$, Takayuki Uchimura ${ }^{1}$, Yasuyuki Kinjo ${ }^{1}$, \\ Midori Murakami ${ }^{1}$, Satoshi Fukumitsu ${ }^{1}$, Kenta Anai ${ }^{1}$, Shigeto Ishikawa ${ }^{1}$, and Kiyoshi \\ Yoshino ${ }^{1}$ \\ ${ }^{1}$ University of Occupational and Environmental Health Japan
}

February 13, 2022

\begin{abstract}
A 41-year-old woman was admitted with hemorrhagic shock with abdominal and right flank pain four days after undergoing cesarean delivery. Contrast-enhanced computed tomography revealed a large retroperitoneal hematoma. Angiography showed a ruptured right ovarian artery aneurysm, anastomosing with a dilated uterine artery. She was successfully treated with transcatheter arterial embolization.
\end{abstract}

\section{Case Report}

Spontaneous postpartum rupture of an ovarian artery aneurysm treated with embolization: A case report and review of the literature.

Mami Shibahara ${ }^{a}$, Emi Kondo ${ }^{a}$, Eiji Shibata ${ }^{a}$, Takayuki Uchimura $^{a}$, Yasuyuki Kinjo ${ }^{a}$, Midori Murakami ${ }^{a}$, Satoshi Fukumitsu ${ }^{\mathrm{b}}$, Kenta Anai ${ }^{\mathrm{b}}$, Shigeto Ishikawa ${ }^{\mathrm{c}}$, and Kiyoshi Yoshino ${ }^{\mathrm{a}}$

${ }^{a}$ Department of Obstetrics and Gynecology, University of Occupational and Environmental Health, Fukuoka, Japan

b Department of Radiology, University of Occupational and Environmental Health, Fukuoka, Japan

c Department of Emergency Medicine, University of Occupational and Environmental Health, Fukuoka, Japan

Corresponding author

Emi Kondo

E-mail:ek89224@med.uoeh-u.ac.jp

1-1 Iseigaoka, Yahatanishi-ku, Kitakyushu, Fukuoka, 807-8555

Telephone: +81-93-603-1611

Fax: +81-93-691-9337

Abstract

A 41-year-old woman was admitted with hemorrhagic shock with abdominal and right flank pain four days after undergoing cesarean delivery. Contrast-enhanced computed tomography revealed a large retroperitoneal 
hematoma. Angiography showed a ruptured right ovarian artery aneurysm, anastomosing with a dilated uterine artery. She was successfully treated with transcatheter arterial embolization.

Key Clinical Message

The spontaneous rupture of an ovarian artery should be considered in patients presenting with flank pain during the peripartum period. Transcatheter arterial embolization is an effective treatment. However, an emergency laparotomy should be prepared.

Introduction

The spontaneous rupture of a perinatal ovarian aneurysm has not been investigated thoroughly. Only 21 cases have been reported in the English literature from 1963 to 2021. ${ }^{1-21}$ All cases have involved multiparas, and the mechanisms of formation and rupture were reportedly related to hemodynamic and hormonal alterations during pregnancy. Ruptured ovarian aneurysms initially present with abdominal pain without genital bleeding. Due to the ubiquity of postpartum abdominal pain, it is difficult to distinguish a sign of rupture from the typical afterpain. A ruptured ovarian aneurysm is a fatal disease, leading to rapid retroperitoneal hemorrhage. The abdominal pain is progressive, and the vital signs of patients progress into a shocked state. The treatment of ruptured ovarian artery aneurysm is laparotomy or transcatheter arterial embolization (TAE), which has been preferred in recent reports. We report the case of a patient, who had spontaneous rupture of an ovarian artery aneurysm and uterine artery aneurysm after undergoing cesarean section (CS) and was successfully treated via TAE. Published case reports involving similar cases were also reviewed.

Case Presentation

A 41-year-old Japanese woman, gravida 2, para 2, gave birth to a healthy baby girl, weighing 2902 g, by elective CS due to a previous CS in the obstetrics general clinic. There were no surgical complications, and $414 \mathrm{~g}$ of blood was lost. She had a history of two myomectomy surgeries, CS 2 years prior, and postpartum hypertensive disorders of pregnancy (HDP) during her first childbirth. She had no history of HDP during pregnancy. Four days following the CS, severe abdominal pain and right flank pain were noted. She had a hemoglobin level of $8.8 \mathrm{~g} / \mathrm{dL}$, blood pressure of $67 / 40 \mathrm{mmHg}$, and pulse rate of $104 \mathrm{bpm}$. She was transferred to another hospital for further examination and treatment. Two units of red blood cells (RBCs) and fresh frozen plasma (FFP) were transfused. Contrast-enhanced computed tomography (CT) of the abdomen and pelvis confirmed a large retroperitoneal hematoma, measuring $18 \times 13 \times 11 \mathrm{~cm}$, surrounding her right kidney (Fig. 1 A). The contrast media extravasated into the retroperitoneum (Fig. 1 B). Threedimensional CT revealed the right ovarian and uterine artery aneurysms (Fig. 2). The patient was referred to our emergency department for TAE. A total of eight units of RBC and 10 units of FFP were administered during the transfer.

On admission, she had a pulse rate of $108 \mathrm{bpm}$ and blood pressure of $87 / 46 \mathrm{mmHg}$. Physical examination revealed muscle guarding pain and a lower abdominal bulge. She had a hemoglobin level of $8.5 \mathrm{~g} / \mathrm{dL}$, platelet count of $1.87 \times 10^{6} / \mu \mathrm{L}$, international normalized ratio of 1.01 , and fibrinogen of $181 \mathrm{mg} / \mathrm{dL}$. The interventional radiology team was consulted regarding the embolization of the ovarian and uterine arteries.

Angiography was immediately initiated. During the procedure, the patient's blood pressure dropped to 70 $\mathrm{mmHg}$, and her pulse disappeared. The patient was immediately intubated. Following tracheal intubation, the patient's vital signs stabilized, and angiography was safely resumed. On angiography, extravascular leakage of the contrast medium from the right ovarian artery, a pseudoaneurysm, and an anastomosis with a dilated uterine artery in the periphery were noted (Fig. $3 \mathrm{~A}, \mathrm{~B}$ ). Selective embolization of the right ovarian artery was performed using n-butyl-2-cyanoacrylate. Although the uterine artery did not exhibit extravasation, it was embolized using gelatin sponge particles to prevent rebleeding of the right ovarian artery through collateral blood flow. Upon completing TAE, 16 units of RBC and 22 units of FFP were transfused (including in the previous hospital).

One day after embolization, the patient was extubated. Her antibiotics were upgraded to sulbactam/ampicillin, and the hemoglobin level and size of the hematoma were monitored. The patient's 
hemoglobin level stopped decreasing after two days. Three days after the embolization, the patient's systolic blood pressure rose and plateaued in the $160 \mathrm{mmHg}$ range. She was diagnosed with postpartum HDP and was treated with nifedipine, an antihypertensive medication. The patient was discharged 17 days after embolization, and her hemoglobin level returned to $12.1 \mathrm{~g} / \mathrm{dL}$.

\section{Discussion}

In decreasing order, the most commonly affected vessels during pregnancy-related rupture of arterial aneurysms are the intracranial arteries, aorta, splenic artery, renal artery, and ovarian artery. ${ }^{22}$ The spontaneous rupture of an ovarian artery aneurysm is rare.

There have only been 21 reports of spontaneous pregnancy-related rupture of an ovarian artery aneurysm in the English literature from 1963 to 2021 [Table 1]. ${ }^{1-21}$ The ages of the patients ranged from 23 to 41 years (mean 34.2 and median 35.5 years). Among patients whose obstetric history was available, all were multigravida (21 cases), including seven grand multiparas (33\%), defined as mothers who have given birth five or more times. There were 18 vaginal deliveries $(82 \%)$ and four CS cases (18\%). The aneurysm occurred on the left in 10 cases and on the right in 12 cases. There were no noted differences related to the laterality of the rupture. In 18 out of 22 cases ( $82 \%$ ), the ruptures occurred during the postpartum period. Specifically, the ruptures occurred within five days after delivery in 17 cases (94\%). The most frequent clinical manifestations included abdominal and flank pain due to retroperitoneal hematoma formation. Since abdominal pain commonly presents during puerperium, it is likely to be underestimated in the absence of active genital bleeding. In the past, ruptured ovarian artery aneurysms were treated via laparotomy. However, most cases have been recently managed via TAE. The present case was the first case involving ovarian and uterine artery aneurysms.

The mechanisms of the formation and rupture of ovarian artery aneurysms were theoretically due to hemodynamic and hormonal alterations during pregnancy. The increased cardiac output and total blood volume due to the uterine blood demand during pregnancy increases mechanical forces on the vessel walls. The increased estrogen and progesterone levels influence arterial wall remodeling, and promote fibromuscular dysplasia of the tunica media and fragmentation of the elastic fibers. These increase the likelihood of aneurysm formation. ${ }^{4,22}$ These alterations have been observed in successive pregnancies, and all previous cases involved multiparas. The patient in our case presented with ovarian and uterine artery aneurysms. The mechanisms of ovarian and uterine artery aneurysm formation were reportedly similar, but no cases of concomitant ovarian and uterine artery aneurysm formation have been reported. Uterine artery pseudoaneurysm (UAP) occurs after traumatic delivery or surgery, such as CS or myomectomy. ${ }^{23,24}$ In the present case, the uterine artery aneurysm likely resulted from the history of CS and myomectomy, as well as the hemodynamic and hormonal changes during pregnancy. Ovarian artery aneurysm ruptures were typically detected later than UAP ruptures because it caused sudden hemorrhagic shock without warning signs, such as genital bleeding. In addition, hypertension was a reported risk factor for the rupture of an ovarian artery aneurysm. ${ }^{25}$ In our case, the patient had a history of HDP, which likely caused pressure on the blood vessels.

The present case showed that retroperitoneal hematomas can be diagnosed via contrast-enhanced CT, and their location can be identified via angiography. In addition, tracheal intubation was required to stabilize the patient's vital signs during angiography. When managing hemodynamically unstable patients, the vital signs and laboratory parameters should be closely monitored during embolization, and an emergency laparotomy should be prepared.

Conclusion

The spontaneous rupture of an ovarian artery aneurysm is rare. This disease should be considered in patients presenting with flank pain during the peripartum period. Early diagnosis and therapy are critical in the management of this fatal disease. Previous cases have identified TAE as an effective treatment option. However, an emergency laparotomy should be prepared. 
Acknowledgments

We are grateful to the patient, who gave her informed consent for publication.

Conflict of interest

None.

Author Contributions

MS conceived the paper and drafted the manuscript. EK and ES provided revisions and drafted portions of the manuscript. TU, YK and MM performed a literature search. SF and KA performed TAE and provided Figure 3 with captions. SI assisted in the preparation of the manuscript. KY revised and finalized the manuscript. All the authors have read and confirmed the final version.

Consent

The authors confirm that the patient consent has been signed and collected in accordance with the journal's patient consent policy.

References

1. Caillouette JC, Owen HW. Postpartum spontaneous rupture of an ovarian-artery aneurysm. Obstet Gynecol. 1963;21:510-511

2. Tsoutsoplides GC. Post-partum spontaneous rupture of a branch of the ovarian artery. Scott Med J. 1967;12:289-290.

3. Riley PM. Letter: Rupture of right ovarian artery aneurysm during delivery. S Afr Med J. 1975;49:729.

4. Burnett RA, Carfrae DC. Spontaneous rupture of ovarian artery aneurysm in the puerperium. Two case reports and a review of the literature. Br J Obstet Gynaecol 1976;83(9):744-750.

5. Jafari K, Saleh I. Postpartum spontaneous rupture of ovarian artery aneurysm. Obstet Gynecol. 1977;49:493-495

6. Mojab K, Rodriguez J. Postpartum ovarian artery rupture with retroperitoneal hemorrhage. AJR Am J Roentgenol. 1977;128:695-696.

7. Høgdall CK, Pedersen SJ, Ovlisen BO, Helgestrand UJ. Spontaneous rupture of an ovarian-artery aneurysm in the third trimester of pregnancy. Acta Obstet Gynecol Scand. 1989;68:651-652.

8. King WL. Ruptured ovarian artery aneurysm: a case report. J Vasc Surg. 1990;12:190-193.

9. Belfort MA, Simon T, Kirshon B, Howell JF. Ruptured ovarian artery aneurysm complicating a term vaginal delivery. South Med J. 1993;86:1073-1074.

10. Guillem P, Bondue X, Chambon JP, Lemaitre L, Bounoua F. Spontaneous retroperitoneal hematoma from rupture of an aneurysm of the ovarian artery following delivery. Ann Vasc Surg. 1999;13:445-448.

11. Blachar A, Bloom AI, Golan G, Venturero M, Bar-Ziv J. Case reports. Spiral CT imaging of a ruptured post-partum ovarian artery aneurysm. Clin Radiol. 2000;55:718-720.

12. Panoskaltsis T, Padwick M, Thomas JM, el Sayed T. Spontaneous rupture of ovarian arterial aneurysm in the antenatal period. Acta Obstet Gynecol Scand. 2000;79:718-719.

13. Kale A, Akdeniz N, Erdemoglu M, Ozcan Y, Yalinkaya A. Spontaneous rupture of the ovarian artery following spontaneous vaginal birth. Saudi Med J. 2005;26:1826-1827.

14. Poilblanc M, Winer N, Bouvier A, Gillard P, Boussion F, Aubé C, et al. Rupture of an aneurysm of the ovarian artery following delivery and endovascular treatment. Am J Obstet Gynecol. 2008;199:e7-8.

15. Mohammed N, Rai H, Koo B, Hoveyda F. Postpartum rupture of ovarian artery. J Obstetrics Gynecol 2011;31(6):548-549.

16. Sakaguchi I, Ohba T, Ikeda O, Yamashita Y, Katabuchi H. Embolization for postpartum rupture of ovarian artery aneurysm: Case report and review. J Obstet Gynaecol Res 2015;41(4):623-627.

17. Wakimoto S, Hidaka N, Fukushima K, Kato K. Spontaneous postpartum rupture of an ovarian artery aneurysm: A case report of successful embolization and a review of the published work. J Obstet Gynaecol Res 2015;41(3):456-459. 
18. Ola PK, Nath RK, Pandit N. Successful management of a rare case of ruptured ovarian artery aneurysm by coil embolization. J Obstet Gynaecol India 2015;65(6):423-425.

19. Enakpene CA, Stern T, Barzallo Salazar MJ, Mukherjee P. Spontaneous rupture of an ovarian artery aneurysm: A rare postpartum complication. Case Rep Obstet Gynecol 2016;1029561:1-4.

20. Stanborough R, Hamrick K, Stephens C. Spontaneous postpartum rupture of bilateral ovarian arteries treated with transarterial embolization. J Obstet Gynaecol Res 2018;44(2):337-340.

21. Yin HF, Liu HY, Jin WT, Hu R. Postpartum spontaneous rupture of an ovarian artery aneurysm: A case report and review. Reprod Dev Med 2020;4(3):191-193.

22. Barrett JM, Van Hooydonk JE, Boehm FH. Pregnancy-related rupture of arterial aneurysms. Obstet Gynecol Surv 1982;37(9):557-66.

23. Kuwata T, Matsubara S, Kaneko Y, Izumi A, Nakata M, Suzuki M. Asymptomatic uterine artery pseudoaneurysm after cesarean section. J Obstet Gynaecol Res 2010;36(2):405-410.

24. Takeda A, Koike W, Imoto S, Nakamura H. Conservative management of uterine artery pseudoaneurysm after laparoscopic-assisted myomectomy and subsequent pregnancy outcome: case series and review of the literature. Eur J Obstet Gynecol Reprod Biol 2014;182:146-153

25. Chao L-W, Chen C-H. Spontaneous rupture of an ovarian artery aneurysm: case report and review of the literature. Gynecol Obstet Invest 2009;68(2):104-107.

Figure legends

Figure. 1

Contrast-enhanced computed tomography image of the abdomen and pelvis

A: A massive hematoma measuring $18 \times 13 \times 11 \mathrm{~cm}$ surrounding her right kidney

B: Dilated right ovarian artery (arrow)

Figure. 2

Three-dimensional computed tomography images showing the aneurysms of the right ovarian artery (transverse arrow) and uterine artery (vertical arrow).

Figure. 3

Angiography

Extravascular leakage of contrast medium from the right ovarian artery with a pseudoaneurysm (arrow A). Anastomosis with a dilated uterine artery in the periphery (B arrow).

\begin{tabular}{|c|c|c|c|c|c|c|}
\hline Case & Age & Gravida/para & Clinical presentation & Side & Onset & Delivery route \\
\hline 1 & 29 & G4P4 & Left flank pain & Left & PPD 2 & VD \\
\hline 2 & 35 & G6P3 & Abdominal pain & Left & PPD 4 & VD \\
\hline 3 & 38 & G6P6 & Abdominal pain and dizziness & Right & During delivery & $\mathrm{VD}$ \\
\hline 4 & 32 & G3P3 & Abdominal pain & Left & PPD 4 & VD \\
\hline 5 & 26 & G5P4 & Abdominal pain and flank pain & Right & PPD 1 & VD \\
\hline 6 & 23 & $\mathrm{~N} / \mathrm{A}$ & Abdominal pain and flank pain & Right & Postpartum month 1 & $\mathrm{VD}$ \\
\hline 7 & 31 & G4P3 & Abdominal pain and fainting & Right & 39 weeks of gestation & CS \\
\hline 8 & 36 & G5P5 & Right flank pain & Right & PPD 4 & $\mathrm{VD}$ \\
\hline 9 & 38 & G3P2 & Right flank pain & Right & During delivery & $\mathrm{VD}$ \\
\hline 10 & 38 & G3P2 & Abdominal pain, meteorism and ileus & Right & PPD 4 & VD \\
\hline 11 & 38 & G12P11 & Right flank pain & Right & PPD 3 & $\mathrm{VD}$ \\
\hline 12 & 37 & G5P4 & Abdominal pain and dyspnea & Left & 39 weeks of gestation & CS \\
\hline 13 & 30 & G5P5 & Abdominal pain, nausea, and vomiting & Left & Postpartum 5 hours & $\mathrm{VD}$ \\
\hline 14 & 39 & G5P4 & Abdominal pain & Right & PPD 5 & VD \\
\hline 15 & 32 & $\mathrm{G} 4 \mathrm{P} 4$ & Abdominal pain & Left & PPD 2 & VD \\
\hline
\end{tabular}




\begin{tabular}{lllllll}
16 & 31 & G6P4 & Abdominal pain & Left & PPD 2 & VD \\
17 & 37 & G4P4 & Abdominal pain & Left & PPD 4 & VD \\
18 & 35 & P3 & Abdominal pain & Left & PPD 3 & VD \\
19 & 38 & P5 & Abdominal pain, weakness, dizziness & Left & PPD 4 & VD \\
20 & 31 & G6P5 & Abdominal pain and flank pain & Right & PPD 5 & VD \\
21 & 38 & G6P2 & Constent low-grade fever & Right & Within PPD 4 & CS \\
22 & 41 & G2P2 & Abdominal pain and flank pain & Right & PPD 4 & CS \\
\hline
\end{tabular}

Table 1: Reported cases of pregnancy-related spontaneous rupture of ovarian artery aneurysm

N/A, not available; PPD, postpartum day; VD, vaginal delivery; CS, cesarean section; LAP, laparotomy; EMB, embolization

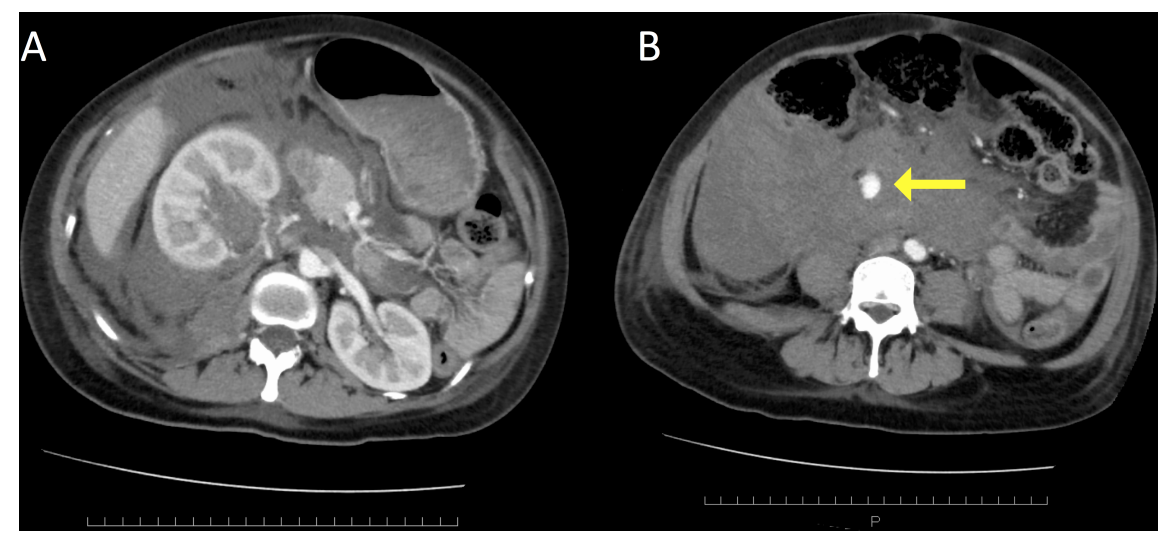




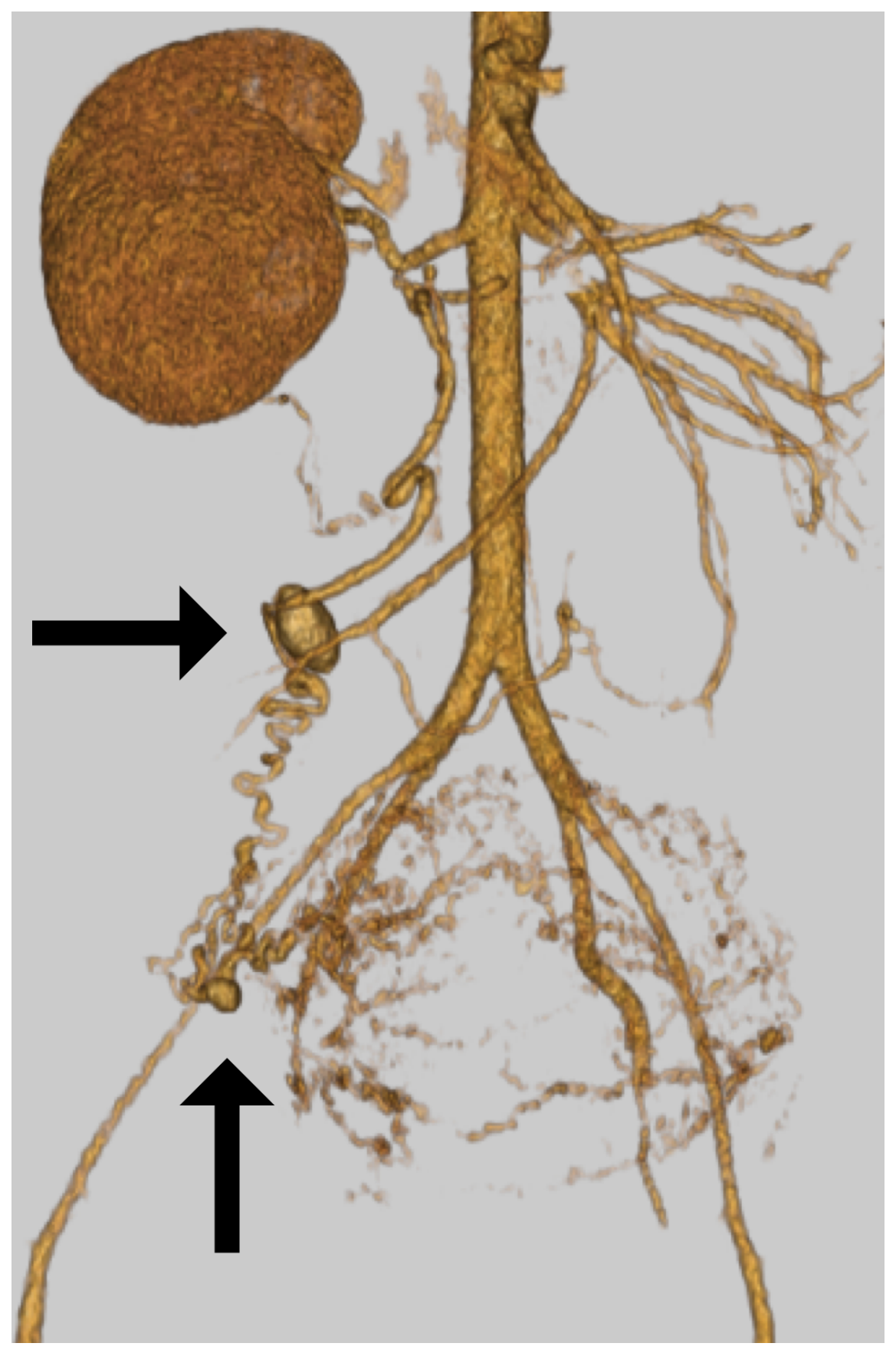




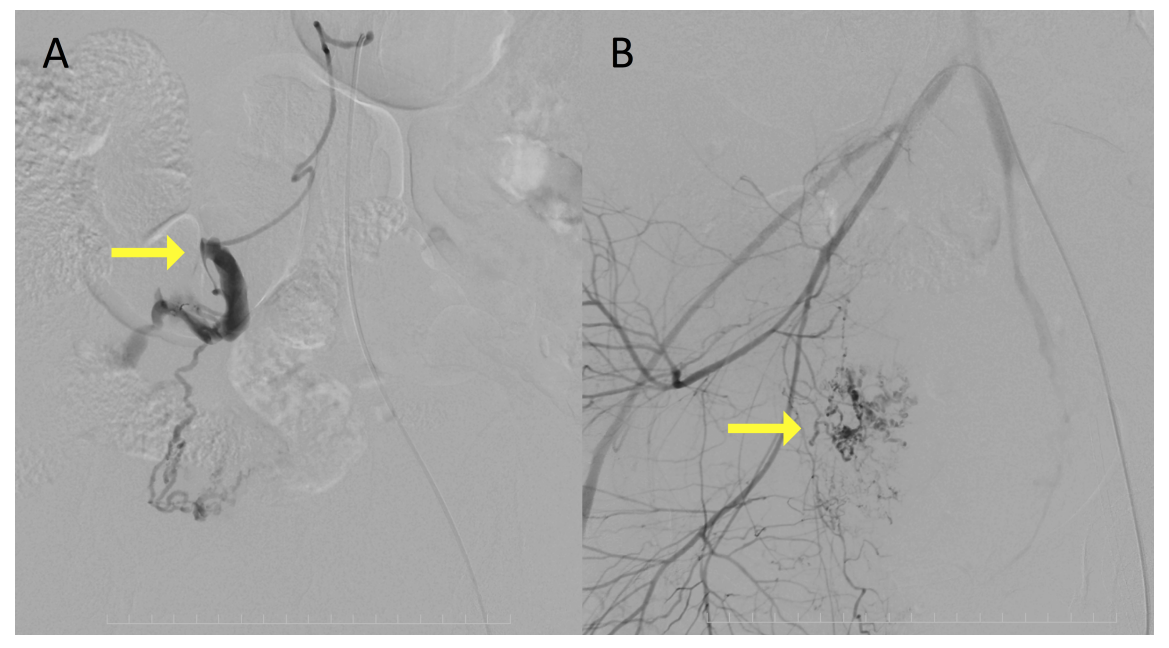

